\title{
Inverse-transformation of 4-dimensional Rindler spacetime
}

\author{
Sangwha-Yi* \\ Department of Math, Taejon University 300-716, South Korea
}

*Corresponding Author: Sangwha-Yi, Department of Math, Taejon University 300-716, South Korea

\begin{abstract}
In special relativity theory, we discovered 4-dimensional transformation of general Rindler spacetime from 4-dimensional Lorentz transformation in inertial frames. We try to discover 4-dimensional inversetransformation of general Rindler space-time.
\end{abstract}

Keywords: Special Relativity theory, Transformation of Rindler space-time; Inverse transformation of Rindler space-time

PACS Number: 03.30. $+p$

\section{INTRODUCTION}

In special relativity theory, we discovered 4-dimensional transformation of general Rindler spacetime from 4-dimensional Lorentz transformation in inertial frames [9]. We try to discover 4dimensional inverse-transformation of general Rindler space-time

At first, 2-dimensional transformation is in Rindler spacetime,

$$
\begin{aligned}
& c t=\left(\frac{c^{2}}{a_{0}}+\xi^{1}\right) \sinh \left(\frac{a_{0} \xi^{0}}{c}\right), x=\left(\frac{c^{2}}{a_{0}}+\xi^{1}\right) \cosh \left(\frac{a_{0} \xi^{0}}{c}\right)-\frac{c^{2}}{a_{0}} \\
& y=\xi^{2}, z=\xi^{3}
\end{aligned}
$$

We know 4-dimensional transformation in Rindler space-time[9],

$$
\begin{aligned}
& c t=\sinh \left(\frac{a_{0} \xi^{0}}{c}\right) \frac{c^{2}}{a_{0}}\left(1+\frac{\vec{a}_{0}}{c^{2}} \cdot \vec{\xi}\right) \\
& \vec{x}=\vec{\xi}+\frac{c^{2}}{a_{0}{ }^{2}} \cosh \left(\frac{a_{0} \xi^{0}}{c}\right) \vec{a}_{0}-\left(1-\cosh \left(\frac{a_{0} \xi^{0}}{c}\right)\right) \frac{\vec{a}_{0} \cdot \vec{\xi}}{a_{0}{ }^{2}} \vec{a}_{0}-\frac{c^{2}}{a_{0}{ }^{2}} \vec{a}_{0}
\end{aligned}
$$

Hence, the proper time is [8]

$$
\begin{aligned}
& d \tau^{2}=d t^{2}-\frac{1}{c^{2}} d \vec{x} \cdot d \vec{x}=d t^{2}-\frac{1}{c^{2}}\left(d x^{2}+d y^{2}+d z^{2}\right) \\
& =\left(1+\frac{\vec{a}_{0} \cdot \vec{\xi}}{c^{2}}\right)^{2}\left(d \xi^{0}\right)^{2}-\frac{1}{c^{2}}\left(\left(d \xi^{1}\right)^{2}+\left(d \xi^{2}\right)^{2}+\left(d \xi^{3}\right)^{2}\right)
\end{aligned}
$$

\section{4-DIMENSIONAL INVERSE-TRANSFORMATION IN RINDLER SPACETIME}

To discover 4-dimensional inverse transformation, if we treat $\frac{\vec{a}_{0}}{c^{2}} \cdot E q(3)$,

$$
\frac{\vec{a}_{0}}{c^{2}} \cdot \vec{x}=\frac{\vec{a}_{0}}{c^{2}} \cdot \vec{\xi}+\cosh \left(\frac{a_{0} \xi^{0}}{c}\right)-\left(1-\cosh \left(\frac{a_{0} \xi^{0}}{c}\right)\right) \frac{\vec{a}_{0} \cdot \vec{\xi}}{c^{2}}-1
$$

Therefore, Eq (5) is 


$$
\frac{c^{2}}{a_{0}}\left(1+\frac{\vec{a}_{0}}{c^{2}} \cdot \vec{x}\right)=\frac{c^{2}}{a_{0}} \cosh \left(\frac{a_{0} \xi^{0}}{c}\right)\left(1+\frac{\vec{a}_{0} \cdot \vec{\xi}}{c^{2}}\right)
$$

$\mathrm{Eq}(2)$ is

$$
c t=\sinh \left(\frac{a_{0} \xi^{0}}{c}\right) \frac{c^{2}}{a_{0}}\left(1+\frac{\vec{a}_{0}}{c^{2}} \cdot \vec{\xi}\right)
$$

Hence, we compare $\mathrm{Eq}(6)$ and $\mathrm{Eq}(7)$, we discover 4-dimensional inverse-transformation in Rindler spacetime.

$$
\frac{c t}{\frac{c^{2}}{a_{0}}\left(1+\frac{\vec{a}_{0} \cdot \vec{x}}{c^{2}}\right)}=\tanh \left(\frac{a_{0} \xi^{0}}{c}\right) \rightarrow \xi^{0}=\frac{c}{a_{0}} \tanh ^{-1}\left\{\frac{c t}{\frac{c^{2}}{a_{0}}\left(1+\frac{\vec{a}_{0} \cdot \vec{x}}{c^{2}}\right)}\right\}
$$

And,

$$
\frac{c^{2}}{a_{0}}\left(1+\frac{\vec{a}_{0} \cdot \vec{\xi}}{c^{2}}\right)=\sqrt{\frac{c^{4}}{a_{0}^{2}}\left(1+\frac{\vec{a}_{0} \cdot \vec{x}}{c^{2}}\right)^{2}-c^{2} t^{2}}
$$

Hence,

$$
\frac{\vec{a}_{0} \cdot \vec{\xi}}{a_{0}}=\sqrt{\frac{c^{4}}{a_{0}^{2}}\left(1+\frac{\vec{a}_{0} \cdot \vec{x}}{c^{2}}\right)^{2}-c^{2} t^{2}}-\frac{c^{2}}{a_{0}}
$$

In this time, if we suppose the condition,

$$
\begin{aligned}
& \vec{a}_{0} \cdot \vec{\xi}=a_{0} \xi \cos \theta, \quad 0 \leq \theta \leq \pi, \theta \neq \frac{\pi}{2}, \\
& |\vec{\xi}|=\xi,\left|\vec{a}_{0}\right|=a_{0}
\end{aligned}
$$

Therefore, we discover 4-dimensional inverse-transformation in Rindler spacetime..

$$
\begin{aligned}
& \xi^{0}=\frac{c}{a_{0}} \tanh ^{-1}\left\{\frac{c t}{\frac{c^{2}}{a_{0}}\left(1+\frac{\vec{a}_{0} \cdot \vec{x}}{c^{2}}\right)}\right\} \\
& \xi=\frac{1}{\cos \theta}\left[\sqrt{\frac{c^{4}}{a_{0}^{2}}\left(1+\frac{\vec{a}_{0} \cdot \vec{x}}{c^{2}}\right)^{2}-c^{2} t^{2}}-\frac{c^{2}}{a_{0}}\right]
\end{aligned}
$$

\section{CONCLUSION}

We know general Rindler coordinate inverse-transformation from 4-dimensional Rindler transformation..

\section{REFERENCES}

[1] S.Yi,"Expansion of Rindler Coordinate Theory and Light's Doppler Effect", The African Review of Physics,8,37(2013)

[2] S.Weinberg,Gravitation and Cosmology(John wiley \& Sons,Inc,1972)

[3] W.Rindler, Am.J.Phys.34.1174(1966)

[4] P.Bergman,Introduction to the Theory of Relativity(Dover Pub. Co.,Inc., New York,1976),Chapter V

[5] C.Misner, K,Thorne and J. Wheeler, Gravitation(W.H.Freedman \& Co.,1973)

[6] S.Hawking and G. Ellis,The Large Scale Structure of Space-Time(Cam-bridge University Press,1973) 
[7] R.Adler,M.Bazin and M.Schiffer,Introduction to General Relativity(McGraw-Hill,Inc.,1965)

[8] Theory of relativity/Rindler coordinates-Wikiversity

[9] S.Yi, "Transformation of 4-dimensional Rindler spacetime",preprint(2020)

Citation: Sangwha-Yi, (2020). Inverse-transformation of 4-dimensional Rindler spacetime. International Journal of Advanced Research in Physical Science (IJARPS) 7(5), pp.31-33 2020.

Copyright: (C) 2020, Authors, This is an open-access article distributed under the terms of the Creative Commons Attribution License, which permits unrestricted use, distribution, and reproduction in any medium, provided the original author and source are credited. 